\title{
Correction: Open science challenges, benefits and tips in early career and beyond
}

\author{
Christopher Allen, David M. A. Mehler
}

There is an error in the reference citations in Table 1 . Reference 24 is incorrectly cited; the correct reference should be 26 .

The citation of references 46 and 47 in the section 'Benefit 1: Greater faith in research' (page 6 of PDF) and in the Fig 1 legend is inappropriate. The correct references are:

Fanelli D. Negative results are disappearing from most disciplines and countries. Scientometrics. 2012;90: 891-904. doi:10.1007/s11192-011-0494-7

Cristea IA, Ioannidis JPA. P values in display items are ubiquitous and almost invariably significant: A survey of top science journals. PLoS One. 2018; 1-15. doi:10.1371/journal. pone.0197440

The citations of reference 46 in the sections 'Challenge 3: Incentive structure isn't in place yet' (page 5 of PDF) and 'Benefit 3: Investment in your future' (page 9 of PDF) are correct.

The citation of reference 47 in the section 'Challenge 3: Incentive structure isn't in place yet' (page 5 of PDF) is correct.

The URL associated with reference 9 is incorrect. The correct URL is: https://www. sciencedirect.com/science/article/pii/B9780128161791000104

There is an error in the reference 31 and in its hyperlink. The correct reference and hyperlink are:

Kerr N. HARKing: -hypnothesizing after the results are known. Personal Soc Psychol Rev. 1998;2: 196-217.

https://doi.org/10.1207/s15327957pspr0203_4

The hyperlinked URL of reference 38 directs to the preprint version of the article. The URL of the published paper is: https://journals.sagepub.com/doi/full/10.1177/2515245918776632? casa_token=UUzRegXa2WAAAAAA\%3AVhPQg9K4OwvuPW64CnGNR5EUacSc6Obb_G_ P4KBFq2wfbv3ayuz9kBxIWBcYE_Uihjb4JysqtRQJ

The URL associated with reference 62 contains an error. The correct URL is: https://papers. ssrn.com/sol3/papers.cfm?abstract_id=2256237

G openaccess

Citation: Allen C, Mehler DMA (2019) Correction: Open science challenges, benefits and tips in early career and beyond. PLoS Biol 17(12): e3000587. https://doi.org/10.1371/journal. pbio.3000587

Published: December 6, 2019

Copyright: @ 2019 Allen, Mehler. This is an open access article distributed under the terms of the Creative Commons Attribution License, which permits unrestricted use, distribution, and reproduction in any medium, provided the original author and source are credited.

\section{Reference}

1. Allen C, Mehler DMA (2019) Open science challenges, benefits and tips in early career and beyond. PLoS Biol 17(5): e3000246. https://doi.org/10.1371/journal.pbio.3000246 PMID: 31042704 